OPEN

SUBJECT AREAS:

NONLINEAR OPTICS

PHYSICS

OPTICAL PHYSICS

STATISTICAL PHYSICS, THERMODYNAMICS AND NONLINEAR DYNAMICS

Received

16 July 2012

Accepted

5 October 2012

Published

25 October 2012

Correspondence and requests for materials should be addressed to

A.D. lasd124@

physics.anu.edu.au)

\section{Spontaneous knotting of self-trapped}

\section{waves}

\author{
Anton S. Desyatnikov' ${ }^{1}$ Daniel Buccoliero' ${ }^{1}$ Mark R. Dennis ${ }^{2} \&$ Yuri S. Kivshar ${ }^{1}$
} 'Nonlinear Physics Centre, Research School of Physics and Engineering, The Australian National University, Canberra ACT 0200,
Australia, ${ }^{2}$ H. H. Wills Physics Laboratory, University of Bristol, Tyndall Avenue, Bristol BS8 ITL, UK.

We describe theory and simulations of a spinning optical soliton whose propagation spontaneously excites knotted and linked optical vortices. The nonlinear phase of the self-trapped light beam breaks the wave front into a sequence of optical vortex loops around the soliton, which, through the soliton's orbital angular momentum and spatial twist, tangle on propagation to form links and knots. We anticipate similar spontaneous knot topology to be a universal feature of waves whose phase front is twisted and nonlinearly modulated, including superfluids and trapped matter waves. he fundamental interplay between topology and dynamics is revealed when knots and links form spontaneously during evolution of a system. This phenomenon is often manifested in material filaments such as agitated strings ${ }^{1}$ and excited molecules ${ }^{2}$. Examples of topologically complex three-dimensional fields, from across the sciences, also manifest knot dynamics. In contrast to material filaments, the whole of space of a knotted field must be filled in a way consistent with any localized knot structure. The idea of Lord Kelvin to describe atoms as vortex knots in ether ${ }^{3}$ has found its continuation in nonlinear field theories in the form of knotted, or, more generally, topological solitons $s^{4}$. Knotted soliton $s^{5,6}$ were introduced as energy minimizers of a three-dimensional nonlinear Lagrangian for a vector order parameter, the latter is similar to pseudo-spin in Bose-Einstein condensates (BECs) ${ }^{7,8}$ and multicomponent superconductors ${ }^{9,10}$, as well as the director field in liquid crystals ${ }^{11,12}$ and electric and magnetic field lines in null-solutions to Maxwell equations ${ }^{13,14}$. Other systems display persistent, tangled defect line structures, such as scroll wave sources in chemical and biological reaction-diffusion systems ${ }^{15}$, localized excitations in bistable metamaterials ${ }^{16}$, knotted disclination lines around colloids in nematic liquid crystals $^{17}$, and quantized vortex filaments in turbulent superfluids ${ }^{18,19}$ and trapped matter waves ${ }^{20-22}$.

The idea of knotted fields has gained renewed interest recently ${ }^{23,24}$, as the role of topology comes to the forefront in a range of physical systems, from knot invariants in statistical mechanics ${ }^{25}$, to topological insulators in condensed matter ${ }^{26}$, and quantum computing ${ }^{27}$. Crucially, experimental techniques are now at a stage where knotted and linked topological defect filaments can be engineered in liquid crystals ${ }^{11,17}$ and laser light ${ }^{28-31}$. In the latter case, similar to a one-component $\mathrm{BEC}^{22}$, the field consists of a complex scalar amplitude, in which the defects are quantized vortices ${ }^{32,33}$-- circulations of energy flow where the intensity is zero and the phase undefined ${ }^{34,35}$. A variety of knotted and linked optical vortices can be synthesized with tailored superpositions of free-space modes ${ }^{28,31}$, successfully implemented in experiments with Gaussian laser beams controlled by holograms ${ }^{29-31}$. However, knot topology appears rare if these superpositions are not chosen carefully ${ }^{36,37}$. A similar approach to the construction of knotted optical fields $s^{28,31}$ was employed for vortex knots in BECs ${ }^{22}$. The striking difference, however, is that vortex knots in BECs undergo essentially nonlinear temporal dynamics due to atomatom interactions, whereas optical vortex knots occur as a result of linear interference and are static in time ${ }^{29-31}$. Knots in BECs with repulsive interatomic interaction disintegrate via series of vortex reconnections ${ }^{22}$.

In the system that we describe, knotting of vortices occurs spontaneously as a rotating, anisotropic soliton ${ }^{38,39}$ propagates in a nonlinear medium. We show that as a radially perturbed soliton propagates, vortex loops ${ }^{40}$ occur in the form of rings perpendicular to the propagation direction. This spontaneous vortex nucleation is a consequence of the nonlinear phase accumulation between the soliton's peak and its tail: phase singularities nucleate if this phase difference reaches the value of $\pi$ during evolution along the optical axis $z$, the analogue of time for a two-dimensional pancake $\mathrm{BEC}^{39}$. If the soliton is elliptic and spinning, these loops are twisted in three-dimensional space, and when the twisting parameter is large enough, the vortices reconnect ${ }^{41,42}$ to form knots and links at the edge of the self-trapped region of the light beam. Such spontaneous knotting is absent from linear light beam propagation ${ }^{36,37}$, and in our system, arises from the soliton's angular momentum ${ }^{43}$ and nonlinearity ${ }^{44}$. We 
demonstrate below how vortex knots appear spontaneously in direct numerical simulations and how they can be explicitly constructed using internal vibrational soliton modes.

\section{Results}

Our starting point is the Nonlinear Schrödinger Equation (NLS) ${ }^{44}$ which describes the dynamics of a slowly varying field envelope $E(x, y, z)$ of a paraxial laser beams in a Kerr-type optical media; in dimensionless form,

$$
i \partial_{z} E+\Delta E+n_{2}(I) E=0,
$$

where the nonlinear part $n_{2}(I)$ of the refractive index $n=n_{0}+n_{2}(I)$ is a function of the normalised light intensity $I=|E|^{2}$, the transverse variables $x$ and $y$ in the Laplacian $\Delta=\partial_{x}^{2}+\partial_{y}^{2}$ are measured in units of a characteristic transverse scale $x_{0}$, the propagation variable $z$ is measured in units of $z_{0}=4 \pi n_{0} x_{0}^{2} / \lambda, \lambda$ is the wavelength of light. Here we consider saturable nonlinearity, $n_{2}(I)=I /(1+I)$, describing photorefractive crystals with $n_{0}=2.35$, as the simplest isotropic model of collapse-free nonlinearity supporting stable solitons ${ }^{45}$. For a typical transverse scale $x_{0}=5 \mu \mathrm{m}$ and green light with $\lambda=$ $532 \mathrm{~nm}$ the propagation unit lenght $z_{0}=1.4 \mathrm{~mm}$. Equation (1) has several integrals of motion, such as optical power, $P=\int|E|^{2} d x d y$, and the orbital angular momentum, $L=\operatorname{Im} \int E^{*}\left(x E_{y}-y E_{x}\right) d x d y$, i.e., $d P / d z=0$ and $d L / d z=0$.

The nonlinear dynamics of laser beams can be understood better when compared with well-known linear self-similar modal beams. For small input power and intensity, $I \ll 1$, Eq. (1) approaches the linear optical regime $n_{2}=0$, with fundamental solution in the form of a radially symmetric diffracting Gaussian beam, $E_{\text {linear }}(r, z)=A$ $\exp \left(i r^{2} / \zeta\right) / \zeta$, here $r^{2}=x^{2}+y^{2}$, and the complex parameter $\zeta=4 z-i w_{0}^{2} / \log \sqrt{2}$. Gaussian beams are characterized by their waist parameter $w_{0}$, i.e. the minimal transverse beam size attained in the focal plane, which defines the half-width at half-maximum (HWHM) of the beam intensity, $w(z)=w_{0} \sqrt{1+3 z^{2} / z_{d}^{2}}, z_{d}=\sqrt{3} w_{0}^{2} / \log 4$ is the diffraction length. The real amplitude $A$ defines optical power, $P=\pi A^{2} \log 2 / 2 w_{0}^{2}$. Figure 1 (a) shows HWHM (black line) of such a beam with $z_{d}=2 \sqrt{3}$ and the waist parameter $w_{0}=2 \sqrt{\log 2}$, indicated by a white double arrow. The color-coded representation of the beam's phase distribution Arg $E$ in Fig. 1(a) shows that during propagation the beam acquires a Gouy phase which does not exceed the value of $\pi^{46}$.

In nonlinear media whose refractive index increases with light intensity, a Gaussian beam induces an effective lens which enhances the focusing: the propagation distance to the focus and the focal spot both decrease, see Fig. 1(b). This is the effect of self-focusing, studied since the invention of lasers ${ }^{44}$. The key to the spontaneous knotting of optical vortices follows from the new observation that quantized phase singularities ${ }^{32,33}$ occur transverse to the propagation direction $^{40}$, as is evident from the phase structure in Fig. 1(b). This is an optical vortex with a circulation of energy in the longitudinal plane, in contrast to Laguerre-Gaussian and similar laser modes ${ }^{34,35}$ and vortex solitons ${ }^{47}$ whose Poynting vector circulates in the transverse plane. The vortex core of the latter follows the longitudinal optical axis; in our case in Fig. 1(b) it is a vortex ring which encircles the beam as a "belt" around its waist.

The spontaneous appearance of a vortex ring during self-focusing can be explained by the nonlinear accumulation of phase by the onaxis high-intensity region of the beam, in comparison with the linear phase accumulation in its low-intensity tails. As soon as the phase difference reaches the value of $\pi$, there appears a point of total destructive interference with zero amplitude and phase defect. The cylindrical symmetry of the beam implies then the spontaneous generation of an unknotted vortex loop in Fig. 1(b).

However, stationary laser beams in self-focusing media, known as bright spatial solitons ${ }^{44,45}$, possess flat phase fronts free of defects, despite having a spatially modulated intensity similar to a Gaussian. When the input power exceeds a threshold value, $P>11.7$ for saturable nonlinearity here, a spatial soliton can be formed; the exact stationary solution to Eq. (1) is given by $E(x, y, z)=R(r) \exp (i k z)$, where $R(r)$ is the real soliton envelope determined numerically and $k$ is the soliton propagation constant parameterising soliton family, $P(k)$. It is the unique spatial profile of the soliton-induced waveguide which guarantees the exact compensation of a natural diffraction (diverging wavefronts) by self-focusing (converging wavefronts) and which leaves the wavefront flat.

A perturbed stable soliton exhibits long-lived oscillations of its intensity profile, studied in the past and often referred to as "soliton breathing" 4 , which break the uniformity of phase fronts. Small perturbations of stable solitons lead to their oscillations during propagation; here we consider a particular type of perturbation preserving soliton power, $P=2 \pi \int R^{2} r d r$,

$$
E(x, y, z=0)=R\left(\sqrt{x^{2} / a^{2}+y^{2} / b^{2}}\right) \exp (i \Theta x y) / \sqrt{a b},
$$

with real widths $a, b$ and the phase-twist parameter $\Theta^{38,39,43}$. The exact soliton envelope is recovered for $a=b=1$ and $\Theta=0$. The initial "stretching" with $a=b \neq 1$ and $\Theta=0$ leads to radial oscillations; Fig. 1(c) demonstrates this process together with the previously unknown features of the field's topological structure, namely the appearance of a regular set of vortex rings.

For an elliptic deformation with $a \neq b$ in Eq. (2), a specific value of $\Theta(a, b)$ can be derived using variational analysis, which corresponds to the so-called spiraling elliptic soliton ${ }^{38}$. Analogous diffracting beams in linear media are described by an exact vortex-free Gaussian solution in the transversely rotating frame ${ }^{43}$. In contrast, as our simulations demonstrate in Fig. 1(d), the spiraling soliton is accompanied by a pair of vortices forming a DNA-like double-helix structure, despite the fact that the field at the entrance to nonlinear medium is free of phase singularities.

The vortex unknots and spirals in Figs. $1(c, d)$ are the necessary elements for more complex topological structures such as links and knots $^{31}$. Evidently, the simultaneous radial stretching of the soliton, as in Fig. 1(c), and its twist, as in Fig. 1(d), reconnect the vortex loops into knots and links. In this general case with $a \neq b$ we treat nonzero $\Theta$ as an independent input parameter, this setup can be realised in experiment with a relatively simple arrangement of cylindrical lenses ${ }^{43}$. Thus we assume independent shaping of the elliptic beam profile and imprinting the twisted wavefront and orbital angular momentum; this approach is also instrumental in suppression of collapse instability for optical ${ }^{38}$ and matter ${ }^{39}$ waves with attractive interatomic interaction.

Exploring this idea we performed large scale computer simulation of the nonlinear dynamics of initially elliptically deformed spatial solitons with twisted phase front and observed spontaneous vortex knotting; the results are illustrated with a particular example in Fig. 2. The parameter domain of Eq. (2) that we studied is 4-dimensional; it includes the fundamental (bell-shaped, radially symmetric $R(r)$ ) soliton family parameterized by optical power $P$, two width parameters for the elliptic transverse stretching of the soliton profile $a$ and $b$, and a parameter $\Theta$ for initial phase twist. Figure 2 shows a sequence of vortex topological structures obtained by varying $\Theta$ while keeping a specific triplet of $P, a, b$ unchanged. We can therefore describe these results in terms of varying the beam twist and its orbital angular momentum.

For small spatial twist we observe elliptically deformed vortex rings, similar to Fig. 1(a). For larger twist the deformation of the vortex rings is stronger, and, as the twist increases, they can reconnect with the formation of Hopf links as in Fig. 2(a), while further increase of $\Theta$ leads to the formation of trefoil vortex knots in Figs. 2(b-d). There are several important features present in all our numerical results. First is the persistent character of vortex knotting: while we could not predict the positions of reconnections giving 

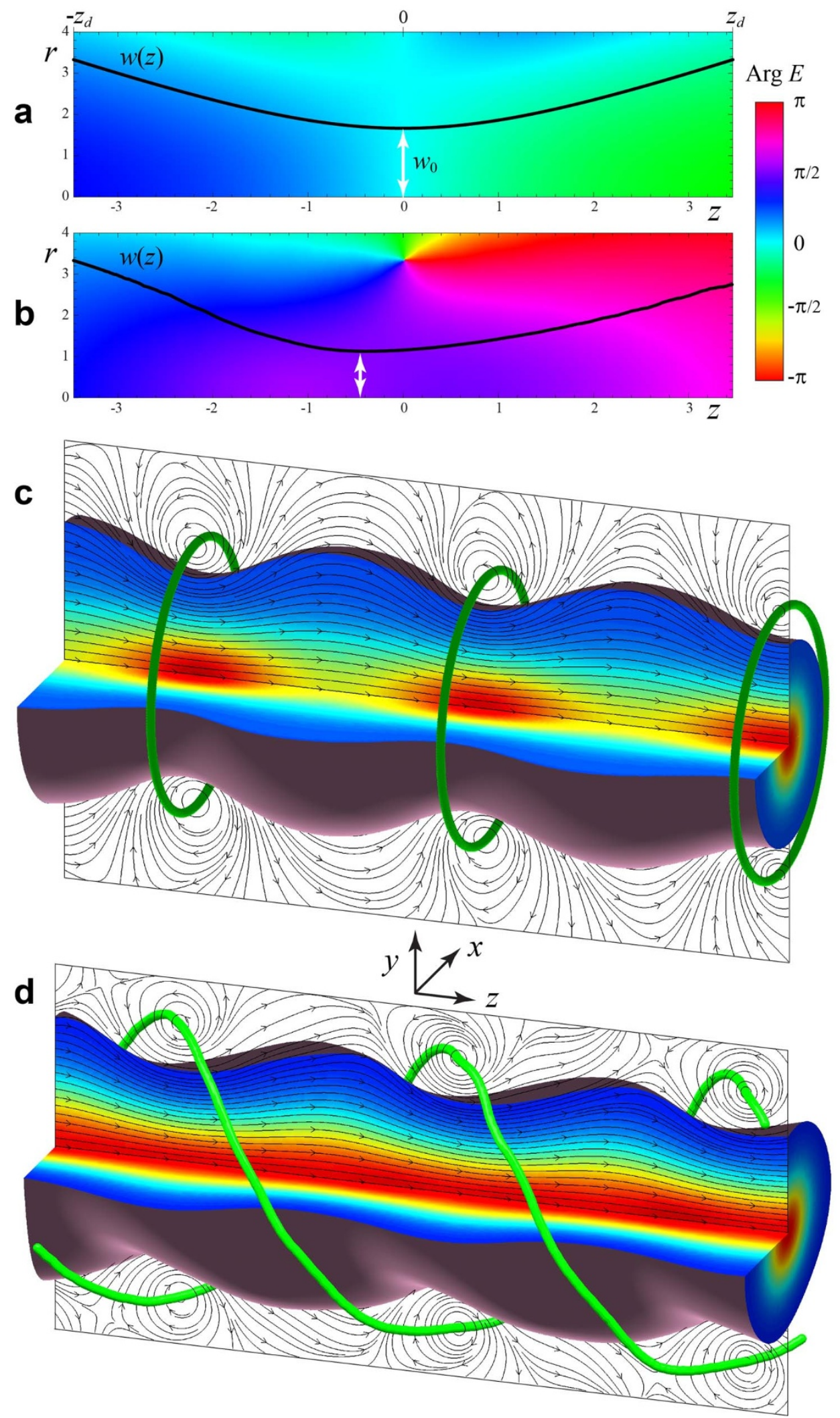

Figure 1 Spontaneous vortex rings and spirals. (a) A Gaussian beam focused in linear media acquires a Gouy phase shift which does not exceed $\pi^{46}$. (b) The same input beam with optical power $P=40$ in a self-focusing saturable medium acquires an additional nonlinear phase difference between the beam's peak on-axis intensity and its tails. When this phase difference reaches a value of $\pi$, a phase singularity appears in the longitudinal plane $(r, z)$; it is seen in (b) as a bright spot at $z \cong 0$ where all colors meet. The corresponding vortex line is a ring (unknot) in the transverse plane ( $x, y$ ). The black solid lines in $(\mathrm{a}, \mathrm{b})$ show the HWHM of intensity $w(z)$ and the white arrows show the positions and the radii of the focal spots (waist parameter). (c) Radial oscillations of a soliton with $k=0.5$ and $P=107$, initially "stretched" with $a=b=1.1$ and $\Theta=0$, see Eq. (2). (d) The elliptic rotating soliton with $P=$ $107, a=1.42, b=0.87$, and $\Theta=0.05^{38}$. The green lines in (c, d) show the position of vortices, the black streamlines with arrows show the Poynting vector in the longitudinal plane $x=0$, and the isosurfaces of intensities are color-coded from small cut-off value (blue) to maximum (red). 

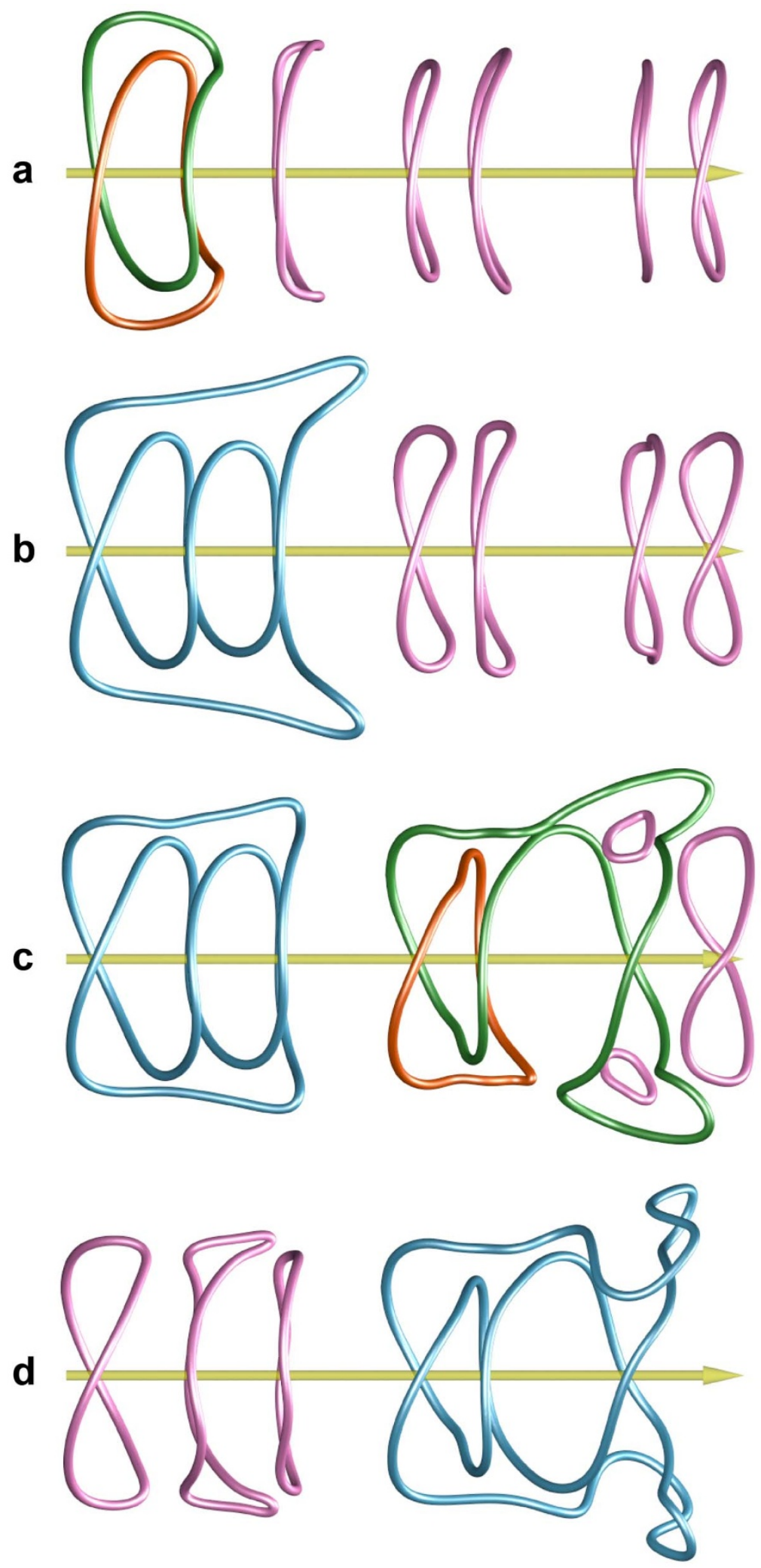

Figure $2 \mid$ Spontaneous vortex links and knots. Initially deformed vortex-free soliton (intensity not shown; $k=0.82, a=1.1, b=1.21$ ) develops vortex lines during propagation in a self-focusing saturable medium (yellow arrow is the optical z-axis, $50 \leq z \leq 150$ ). Here and below in Fig. 3 we distinguish isolated vortex rings (unknots) in pink, linked vortex rings (Hopf links) in green and orange, and vortex (trefoil) knots in blue. For a small initial twist $\Theta$ there are seven isolated vortex rings similar to Fig. 1(c) (not shown), and the increase to $\Theta=0.005$ leads to the appearance of a vortex link in (a) formed by two left rings. Stronger initial twist of $\Theta=0.01$ transforms this link and the third ring into a knot in (b). While this knot is only slightly deformed in (c) with $\Theta=0.013$, the three neighbouring rings reconnect into a link. This link and the remaining right ring in (c) form a knot in $(d)$ with $\Theta=0.015$. At the same time, the left knot in (b, c) disintegrates into three loops in (d). 

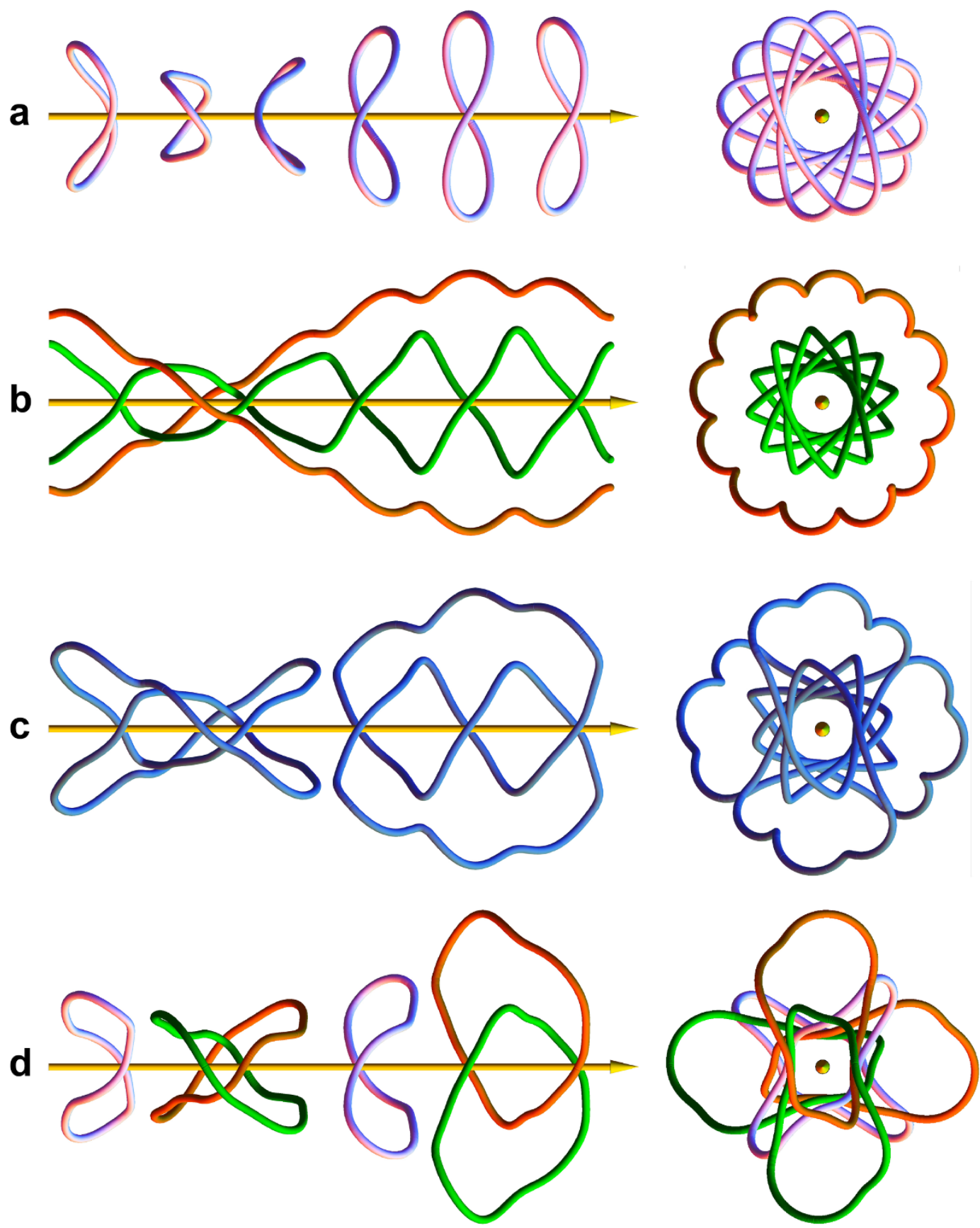

Figure 3 Vortex knots from vibrational modes of a soliton. Vortex lines are shown for different superpositions of the soliton with $k=0.8$ with its monopole and quadrupole modes, see Eqs. (3) and (4). In all graphs, the propagation interval (yellow arrow) $9<z<107$ corresponds to one period $2 \pi /\left(\omega_{0}-\omega_{2}\right) \cong 97.7$, and the amplitude of the monopole mode with $s=0$ and $\omega_{0}=0.383$ equals $\epsilon_{0}=0.1$. The two amplitudes of the quadrupole mode with $\omega_{2}=0.318$ are given by $\epsilon_{1,2}=\varepsilon_{1,2} \exp (i \pi / 4)$ with: (a) $\varepsilon_{1}=0.1, \varepsilon_{2}=0$; (b) $\varepsilon_{1}=0.15, \varepsilon_{2}=0$; (c) $\varepsilon_{1}=0.15, \varepsilon_{2}=0.015$; and (d) $\varepsilon_{1}=0.15$, $\varepsilon_{2}=0.045$.

specific links or knots, they appear in a significant parameter domain where the orbital angular momentum and spatial twist are sufficiently large. Secondly, the links and knots look irregular, which might be a consequence of a symmetry-breaking instability seeded in our simulations by numerical noise. This feature is apparent in Fig. 2(c) and Supplementary Video with a clearly asymmetric Hopf link. Finally, the vortex knots appear to be robust with respect to small change of parameters, e.g. note the smoothly deformed knot in Figs. 2(b, c) which, nevertheless, disintegrates into a triplet of vortex rings with further increase of twist in Fig. 2(d).

\section{Discussion}

The results of numerical simulations in Fig. 2 do not directly provide insight into any possible physical mechanism of vortex knotting because of the spontaneous and irregular character of this process. The basic cause of vortex generation, however, must be the destructive interference between the soliton and the dispersive linear waves radiated during oscillations. The later can be analysed in terms of the dynamics of small perturbations of solitons, e.g. introduced by deformations described by Eq. (2), using Lyapunov stability analysis ${ }^{48}$. Namely, we consider a perturbed soliton in the form 
$E(x, y, z)=e^{i k z}$

$$
\left\{R(r)+u_{s}(r)\left(\epsilon_{1} e^{i s \varphi}+\epsilon_{2} e^{-i s \varphi}\right) e^{i \omega_{s} z}+w_{s}(r)\left(\epsilon_{1} e^{-i s \varphi}+\epsilon_{2} e^{i s \varphi}\right) e^{-i \omega_{s} z}\right\}^{(3)}
$$

with azimuthal angle $\varphi=\tan ^{-1} y / x$ and perturbation mode envelopes $\left|u_{s}\right| \ll R$ and $\left|w_{s}\right| \ll R$, which allow to linearize Eq. (1) and solve it numerically as an eigenvalue problem, recovering the real eigenvector $\left\{u_{s}, w_{s}\right\}$ and eigenfrequency $\omega_{s}$ for each (integer) azimuthal mode index s. Choosing as normalization for the linear modes $\max \left(\left|u_{s}\right|\right.$, $\left.\left|w_{s}\right|\right)=\max (R)$ the moduli $\varepsilon_{1,2}=\left|\epsilon_{1,2}\right|$ of the two arbitrary complex perturbation amplitudes $\epsilon_{1,2}$ can be used as parameters indicating relative strength of the perturbation, $\varepsilon_{1,2} \ll 1$.

Optical vortices are lines in space $\left\{r_{*}, \varphi_{*}, z_{*}\right\}$ on which the field in Eq. (3) vanishes, $E\left(r_{*}, \varphi_{*}, z_{*}\right)=0$. For example, the excitation with radial mode $s=0$ (and a single amplitude $\epsilon_{0}=\epsilon_{1}+\epsilon_{2}$ ) requires solving

$$
C_{1}\left(r_{*}, z_{*}\right)=R\left(r_{*}\right)+\epsilon_{0} u_{0}\left(r_{*}\right) e^{i \omega_{0} z_{*}}+\epsilon_{0} w_{0}\left(r_{*}\right) e^{-i \omega_{0} z_{*}}=0,
$$

so that each solution $\left\{r_{*}, z_{*}\right\}$ is a vortex unknot, as in Figs. 1(b,c). More insight can be gained by noticing that for vanishing amplitude $\varepsilon_{0}=\left|\epsilon_{0}\right| \rightarrow 0$ the solution is diverging, $r_{*} \rightarrow \infty$, i.e. we recover unperturbed vortex-free soliton. For large $r \rightarrow \infty$ the asymptotes of the modes are $\left\{u_{0}, w_{0}\right\} \sim \exp \left(-r \omega_{ \pm}\right) / r \omega_{ \pm}$, here $\omega_{ \pm}^{2}=k \pm \omega_{0}$ with the plus sign for $u_{0}$ and minus sign for $w_{0}$. It follows that we can neglect $u_{0} \ll w_{0}$ and obtain the positions of vortex unknots at $z_{*}=(\delta+2 \pi n) /$ $\omega_{0}$ and their radius $r_{*}, R\left(r_{*}\right)+\varepsilon_{0} w_{0}\left(r_{*}\right)=0$, here $\delta=\operatorname{Arg} \epsilon_{0}$ and integer $n=0, \pm 1, \ldots$. Such analysis gives excellent agreement with the numerical results in Fig. 1(c), although there is no clear mapping between the amplitude $\varepsilon_{0}$, which determines the unknot radii $r_{*}$, and the perturbation strength in Eq. (2).

A similar analysis for $s=2$ in Eq. (3) allows the recovery, for $\epsilon_{1}=0$, of the vortex spirals as in Fig. 1(d), while for $\epsilon_{2}=0$ we obtain vortex spirals of the opposite handedness. We conclude that the radial oscillations in Fig. 1(c) can be excited by a monopole mode with $s$ $=0$ while the spiraling elliptic soliton in Fig. 1(d) bifurcates from a quadrupole mode with $s=2$. The simultaneous action of these two modes, demonstrated in Fig. 3, explains vortex knotting.

To describe the simultaneous elliptic stretching and phase twist employed in the direct numerical simulations in Eq. (3) and Fig. 2, we take the superposition of a soliton and two modes with indices $s=0,2$ in Eq. (3) and derive the quadratic equation $E\left(r_{*}, \varphi_{*}, z_{*}\right)=0$ for $t=$ $e^{i 2 \varphi}$,

$$
C_{2}\left(r_{*}, z_{*}\right) t^{2}+C_{1}\left(r_{*}, z_{*}\right) t+C_{0}\left(r_{*}, z_{*}\right)=0,
$$

where $C_{2,0}\left(r_{*}, z_{*}\right)=\epsilon_{1,2} u_{2}\left(r_{*}\right) e^{i \omega_{2} z_{*}}+\epsilon_{2,1} w_{2}\left(r_{*}\right) e^{-i \omega_{2} z_{*}}$. Among the continuous set of roots of (4) in the plane $\left\{r_{*}, z_{*}\right\}$ we choose only the roots with $|t|=1$; it follows from the definitions of $C_{0,1,2}\left(r_{*}, z_{*}\right)$ that only a single such root can be found at a particular location $\left\{r_{*}\right.$, $\left.z_{*}\right\}$. This root defines a closed or open contour in the plane $\left\{r_{*}, z_{*}\right\}$ which generates a torus by rotation around $z *$ axis - this is the torus on which all vortex lines lie. The root gives two vortices on a torus, $\varphi_{1}=\frac{1}{2} \operatorname{Arg} t$ and $\varphi_{2}=\pi+\varphi_{1}$. Therefore, in each transverse plane $z=$ $z *$ we can obtain either no vortices (no roots with $|t|=1$ in Eq. (4)), or a pair of vortices (open contour in $\left\{r_{*}, z_{*}\right\}$ ), or a quadruplet of vortices (closed contour in $\left\{r_{*}, z_{*}\right\}$ ). The symmetry of the vortex pairs $\varphi_{*} \leftrightarrow \pi$ $+\varphi *$ is a natural consequence of the elliptic perturbation.

For a soliton with given power $P$, with the modal eigenfrequencies $\omega_{0,2}$ and eigenvectors $\left\{u_{0,2}(r), w_{0,2}(r)\right\}$ determined numerically, the key parameters which define the field's topological structure are the relative complex amplitudes $\epsilon_{0,1,2}$ of the internal modes with azimuthal indices $s=0$ and 2 . Varying $\epsilon_{0,1,2}$ we were able to reconstruct all the types of vortex structures observed in direct numerical simulations in Figs. 1 and 2, including vortex rings, spirals, links, and knots. Since the symmetry-breaking instability and the additional noise are absent here, the obtained structures preserve the elliptic symmetry of rotation by $\pi$ around optical axis. Similar to the conclusions drawn from Figs. 1 and 2 we notice here that the domination of the radial mode, $\left|\epsilon_{0}\right| \gg\left|\epsilon_{1,2}\right|$, leads to the vortex unknots in Fig. 3(a), while in the opposite case the vortex spirals appear in Fig. 3(b). The result of combined action of both modes, with the interplay between radial stretching $\left|\epsilon_{0}\right|$ and elliptic twist $\left|\epsilon_{1,2}\right|$ perturbations, is the vortex links and knots in Figs. 3(c,d).

For a given example with $k=0.8$ in Fig. 3 we constructed the lowest order nontrivial vortex topologies: Hopf links and trefoil knots. Additional analysis shows that the order of torus knots that can be constructed is limited by the interplay of two eigenfrequencies in the system: the pulsation rate $\omega_{0}$ and the rotation rate $\omega_{2}$, both of which are determined by the soliton power $P(k)$. We found that the beating of modes with these two frequencies determines the vortex reconnection events and thus the maximal length of the tori in $z$ direction. Taking $k=0.6$ and $P=198$ with the beating period $2 \pi /\left(\omega_{0}\right.$ $\left.-\omega_{2}\right) \cong 209.4$ as an alternative example and varying perturbation amplitudes $\epsilon_{0,1,2}$ we were able to construct the cinquefoil knots $(5,2$ torus knots) and double links (4,2 torus links), in addition to their lower order counterparts in Figs. 3(c,d), i.e. trefoil knots and Hopf links. The full analysis of the parameter domain of Eq. (4) where the knotting occurs is not a straightforward task; we plan to address this open question in future work.

In the general case of an arbitrarily perturbed soliton, complex and quite irregular bundles of vortices appear on soliton tails, not unlike the random vortex tangles in superfluids ${ }^{18,19}$ and speckle fields ${ }^{36,37}$. In other words, many higher-order internal modes are excited simultaneously and their interference complicates the picture. In contrast to the linear waves, however, here the reconnection of vortices and the spontaneous formation of vortex links and knots are governed by the nonlinear dynamics of the self-trapped elliptic beam, i.e. the linear perturbation modes and their frequencies in such superpositions can be controlled in experiment by changing optical power $P$. Therefore, the spontaneous knotting described here does not lead to isolated and ordered knots, as in Refs. ${ }^{29-31}$. Nevertheless, two key experimental parameters can be well controlled: the nonlinearity, by changing the power $P$, and the spiraling rate and orbital angular momentum, by changing the initial phase twist $\Theta^{38,43}$. This approach can be also extended to nonlinear dynamics of random waves, such as speckle fields in optics, as well as development of topological structures in modulationally unstable waves.

The principal role of the spatial twist and orbital angular momentum for spontaneous knotting of nonlinear waves, demonstrated here, may be also important in quantum turbulence in superfluids ${ }^{18}$, consisting of a very complex, apparently disordered, dynamic tangle of moving and reconnecting quantized vortex filaments. The vortex tangle is usually characterized in terms of its density (length of vortex line per unit volume), related to energy. This measure does not take into account, however, the intrinsic disorder, coiling and linking, which occurs within the turbulent vortex tangle. It became clear ${ }^{19}$ that new measures to describe the geometrical and topological complexity of superfluid turbulence are necessary, in particular those available from modern geometry and knot theory, including the existence of quantized vortex knot solutions $^{49}$ and their nonlinear dynamics ${ }^{22,50}$. Another interesting analogy can be drawn with hadronic superfluids in dense nuclear matter where knots may form in neutron superfluid due to the AndreevBashkin effect ${ }^{51,52}$.

1. Raymer, D. M. \& Smith, D. E. Spontaneous knotting of an agitated string. Proc. Natl. Acad. Sci. USA 108, 16432-16437 (2007).

2. Forgan, R. S., Sauvage, J.-P. \& Stoddart, F. J. Chemical Topology: Complex Molecular Knots, Links, and Entanglements. Chem. Rev. 111, 5434-5464 (2011).

3. Thomson, W. H. (Lord Kelvin) On Vortex Atoms. Proc. R. Soc. Edinburgh VI, 94-105 (1867).

4. Manton, N. S. \& Sutcliffe, P. M. Topological Solitons. (Cambridge Monographs on Mathematical Physics, Cambridge University Press, 2004). 
5. Faddeev, L. \& Niemi, A. J. Stable knot-like structures in classical field theory Nature 387, 58 (1997).

6. Battye, R. A. \& Sutcliffe, P. M. Knots as stable soliton solutions in a threedimensional classical field theory. Phys. Rev. Lett. 81, 4798-4801 (1998).

7. Babaev, E., Faddeev, L. D. \& Niemi, A. J. Hidden symmetry and knot solitons in a charged two-condensate Bose system. Phys. Rev. B 65, 100512(R) (2002).

8. Kawaguchi, Y., Nitta, M. \& Ueda, U. Knots in a Spinor Bose-Einstein Condensate. Phys. Rev. Lett. 100, 180403 (2008).

9. Babaev, E. Dual Neutral Variables and Knot Solitons in Triplet Superconductors. Phys. Rev. Lett. 88, 177002 (2002).

10. Garaud, J., Carlstrom, J. \& Babaev, E. Topological Solitons in Three-Band Superconductors with Broken Time Reversal Symmetry. Phys. Rev. Lett. 107, 197001 (2011).

11. Lapointe, C., Mason, T. \& Smalyukh, I. I. Shape-controlled colloidal interactions in nematic liquid crystals. Science 326, 1083-1086 (2009).

12. Alexander, G. P., Chen, B. G., Matsumoto, E. A. \& Kamien, R. D. Disclination Loops, Point Defects, and All That in Nematic Liquid Crystals. Rev. Mod. Phys. 84, 497 (2012).

13. Irvine, W. T. M. \& Bouwmeester, D. Linked and knotted beams of light. Nat. Phys. 4, 716-720 (2008).

14. Arrayás, M. \& Trueba, J. L. Exchange of helicity in a knotted electromagnetic field. Ann. Phys. 524, 71-75 (2012).

15. Winfree, A. T. Persistent tangled vortex rings in generic excitable media. Nature 371, 233-236 (1994).

16. Rosanov, N. N., Vysotina, N. V., Shatsev, A. N., Desyatnikov, A. S. \& Kivshar, Yu. S. Knotted Solitons in Nonlinear Magnetic Metamaterials. Phys. Rev. Lett. 108, 133902 (2012)

17. Tkalec, U., Ravnik, M., Čopar, S., Žumer, S. \& Muševič, I. Reconfigurable Knots and Links in Chiral Nematic Colloids. Science 333, 62 (2011).

18. Quantized vortex dynamics and superfluid turbulence. Eds. C. F. Barenghi, R. J. Donnelly, and W. F. Vinen, Lecture Notes in Physics Vol. 571 (2001).

19. Poole, D. R., Scoffield, H., Barenghi, C. F. \& Samuels, D. C. Geometry and topology of superfluid turbulence. J. Low Temp. Phys. 132, 97-117 (2003).

20. Matthews, M. R., Anderson, B. P., Haljan, P. C., Hall, D. S., Wieman, C. E. \& Cornell, E. A. Vortices in a Bose-Einstein condensate. Phys. Rev. Lett. 83, 2498-2501 (1999)

21. Ruostekoski, J. \& Dutton, Z. Engineering vortex rings and systems for controlled studies of vortex interactions in Bose-Einstein condensates. Phys. Rev. A 72, 063626 (2005)

22. Proment, D., Onorato, M. \& Barenghi, C. F. Vortex knots in a Bose-Einstein condensate. Phys. Rev. E 85, 036306 (2012).

23. Knots and Applications, Intensive Research Period at the Centro di Ricerca Matematica Ennio De Giorgi, Scuola Normale Superiore, Pisa, Italy, 1 May - 31 July (2011).

24. Knotted Fields, research program at the Kavli Institute for Theoretical Physics, University of California, Santa Barbara, USA, 18 June - 14 July (2012).

25. Wu, F. Y. Knot theory and statistical mechanics. Rev. Mod. Phys. 64, 1099-1131 (1992).

26. Hasan, M. Z. \& Kane, C. L. Colloquium: Topological insulators. Rev. Mod. Phys. 82, 3045 (2010)

27. Collins, G. P. Computing with Quantum Knots. Sci. Am. 294, 57 (2006)

28. Berry, M. V. \& Dennis, M. R. Knotted and linked phase singularities in monochromatic waves. Proc. R. Soc. A 457, 2251-2263 (2001).

29. Leach, J., Dennis, M. R., Courtial, J. \& Padgett, M. J. Knotted threads of darkness. Nature 432, 165 (2004).

30. Leach, J., Dennis, M. R., Courtial, J. \& Padgett, M. J. Vortex knots in light. New J. Phys. 7, 55 (2005)

31. Dennis, M. R., King, R. P., Jack, B., O’Holleran, K. \& Padgett, M. J. Isolated optical vortex knots. Nature Physics 6, 118-121 (2010).

32. Nye, J. F. \& Berry, M. V. Dislocations in wave trains. Proc. R. Soc. A 336, 165-190 (1974).

33. Nye, J. F. Natural Focusing and Fine Structure of Light (Inst. Phys. Publ., 1999).

34. Soskin, M. S. \& Vasnetsov, M. V. Singular optics. Prog. Opt. 42, 219 (2001).

35. Dennis, M. R., O'Holleran, K. \& Padgett, M. J. Singular optics: Optical vortices and polarization singularities. Prog. Opt. 53, 293-363 (2009).

36. O'Holleran, K., Dennis, M. R. \& Padgett, M. J. Topology of light's darkness. Phys. Rev. Lett. 102, 143902 (2009).
37. Padgett, M. J., O'Holleran, K., King, R. P. \& Dennis, M. R. Knotted and tangled threads of darkness in light beams. Contemp. Phys. 52, 265 (2011).

38. Desyatnikov, A. S., Buccoliero, D., Dennis, M. R. \& Kivshar, Yu. S. Suppression of collapse for spiraling elliptic solitons. Phys. Rev. Lett. 104, 053902 (2010).

39. Abdullaev, J., Desyatnikov, A. S. \& Ostrovskaya, E. A. Suppression of collapse for matter waves with orbital angular momentum. J. Opt. 13, 064023 (2011).

40. Wright, F. J. \& Berry, M. V. Wave-front dislocations in the soundfield of a pulsed circular piston radiator. J. Acoust. Soc. Am. 75, 733 (1984).

41. Berry, M. V. \& Dennis, M. R. Topological events on wave dislocation lines: Birth and death of small loops, and reconnection. J. Phys. A 40, 65-74 (2007).

42. Berry, M. V. \& Dennis, M. R. Reconnections of wave vortex lines. Eur. J. Phys. 33, $723(2012)$

43. Courtial, J., Dholakia, K., Allen, L. \& Padgett, M. J. Gaussian beams with very high orbital angular momentum. Opt. Commun. 144, 210-213 (1997).

44. Kivshar, Yu, S. \& Agrawal, G. P. Optical Solitons: From Fibers to Photonic Crystals (Academic, 2003).

45. Segev, M., Crosignani, B., Yariv, A. \& Fischer, B. Spatial solitons in photorefractive media. Phys. Rev. Lett. 68, 923-926 (1992).

46. Boyd, R. W. Intuitive explanation of the phase anomaly of focused light beams. J. Opt. Soc. Am. 70, 877 (1980).

47. Desyatnikov, A. S., Kivshar, Yu, S. \& Torner, L. Optical vortices and vortex solitons. Prog. Opt. 47, 291-391 (2005).

48. Yang, J. Internal oscillations and instability characteristics of $(2+1)$-dimensional solitons in a saturable nonlinear medium. Phys. Rev. E 66, 026601 (2002).

49. Samuels, D. C., Barenghi, C. F. \& Ricca, R. L. Quantized Vortex Knots. J. Low Temp. Phys. 110, 509 (1998).

50. Ricca, R., Samuels, D. C. \& Barenghi, C. F. Evolution of vortex knots. J. Fluid Mech. 391, 29 (1999).

51. Babaev, E. Unconventional Rotational Responses of Hadronic Superfluids in a Neutron Star Caused by Strong Entrainment and a $\Sigma$ - Hyperon Gap. Phys. Rev. Lett. 103, 231101 (2009).

52. Babaev, E. Andreev-Bashkin effect and knot solitons in an interacting mixture of a charged and a neutral superfluid with possible relevance for neutron stars. Phys. Rev. D 70, 043001 (2004).

\section{Acknowledgments}

This work is supported by the Australian Research Council and the National Science Foundation under Grant No. NSF PHY11-25915. ASD is an Australian Research Fellow and MRD is a Royal Society University Research Fellow. The authors are grateful to R. King for the help with figure 2. ASD and MRD thank for hospitality the Kavli Institute for Theoretical Physics at University of California in Santa Barbara where this work was completed.

\section{Author contributions}

ASD and MRD suggested the idea in Fig. 1, ASD performed linear stability analysis in Fig. 3 and wrote the paper; DB performed extensive series of numerical simulations in Fig. 2 MRD contributed to the development of numerical algorithms, analysis of the results, and manuscript preparation; YSK supervised the work and contributed to the manuscript preparation. All authors reviewed the manuscript.

\section{Additional information}

Supplementary information accompanies this paper at http://www.nature.com/ scientificreports

Competing financial interests: The authors declare no competing financial interests.

License: This work is licensed under a Creative Commons

Attribution-NonCommercial-NoDerivative Works 3.0 Unported License. To view a copy of this license, visit http://creativecommons.org/licenses/by-nc-nd/3.0/

How to cite this article: Desyatnikov, A.S., Buccoliero, D., Dennis, M.R. \& Kivshar, Y.S. Spontaneous knotting of self-trapped waves. Sci. Rep. 2, 771; DOI:10.1038/srep00771 (2012) 